s.

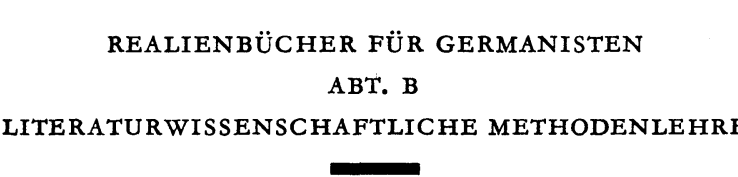




\title{
Die schriftliche Form germanistischer Arbeiten
}

\author{
Empfehlungen \\ für die Anlage und die äußere Gestaltung \\ wissenscbaftlicher Manuskripte \\ unter besonderer Berücksichtigung der \\ Titelangaben von Schrifttum \\ Mit einem Geleitwort von \\ HANS-EGON HASS
}

4., durchgesehene Auflage

MCMLXVI

J.B. METZLERSCHE VERLAGSBUCHHANDLUNG STUTTGART 
I. Auflage 1962 (I.-4. Tsd)

2. Auflage I962 (5.-9. Tsd)

3. Auflage 1964 (IO.-I5. Tsd)

4. Auflage 1966 (I6.-2I. Tsd)

\section{I3}

ISBN 978-3-476-99251-2

ISBN 978-3-476-99250-5 (eBook)

DOI $10.1007 / 978-3-476-99250-5$

(C) Springer-Verlag GmbH Deutschland 1962

Ursprünglich erschienen bei J. B. Metzlersche Verlagsbuchhandlung und Carl Ernst Poeschel Verlag GmbH in Stuttgart 1962 


\section{Geleitwort}

Nur zu wissen, daß es eine Schrift dieses Titels überhaupt gibt, ist für den jungen Studenten schon förderlich. Denn er wird dann nicht mehr ganz so unbefangen nach seiner Willkür verfahren, wie der Anfänger es meist tut. Er wird darauf hingewiesen, daß es bestimmte Regeln, zweckmäßige Verfahrensweisen gibt, nach denen er sich richten sollte. Dieses Bewußtsein allein bedeutet schon einen Schritt über die schlimmste Ahnungslosigkeit hinaus, die leider nicht nur in Anfängerarbeiten so oft festzustellen ist und von dem Dozenten in allen Einzelheiten nur durch einen gewiß nicht zumutbaren Aufwand an Zeit und Mühe zu korrigieren wäre.

Das Wichtigste ist natürlich, sich die erforderliche Belehrung nun auch verschaffen zu können. Bisher mußte der Anfänger selbst mühsam und oft genug ein wenig ratlos nach gültigen Mustern suchen. Denn derartige technische Fragen können von gelegentlichen Hinweisen abgesehen - nicht Thema des akademischen Unterrichts sein. Um diese Lücke auszufüllen, stellte der Verfasser der vorliegenden Schrift zunächst für die germanistischen Tutor-Gruppen an der Freien Universität Berlin die wichtigsten und notwendigsten Regeln zusammen. $\mathrm{Daß}$ sie nun hier vervollständigt und für jeden zugänglich vorliegen, werden hoffentlich viele Studierende der Germanistik dankbar begrüßen; denn nicht als eine Zwangsjacke, sondern als Entlastung von zeitraubender Suche und somit als Hilfe für eine freiere Bewegung sollten diese Regeln aufgefaßt werden.

Ich finde es besonders verdienstvoll, daß der Verfasser sich so viele Probleme hat einfallen lassen, vor die sich der Anfänger gestellt sehen kann, die dem Fortgeschrittenen aber gar nicht mehr als solche bewußt sind. Diese Reichhaltigkeit der Anweisungen sowohl im scheinbar Selbstverständlichen und, wie man glauben könnte, allgemein Bekannten als auch im Entlegenen sollte nicht als skurrile und überflüssige, sondern als 
höchst wünschenswerte und notwendige Pedanterie angesehen werden. Man dürfte sich mit Versen Goethes dazu bekennen:

Das mach' ich mir denn zum reichen Gewinn,

$\mathrm{Daß}$ ich getrost ein Pedante bin.

So scheint mir dieser Band der 'Sammlung Metzler sehr willkommen als nützliche Fibel für den Anfänger wie auch als bequemer gelegentlicher Ratgeber für den Fortgeschrittenen. Darüber hinaus möchte ich der Schrift aber noch den besonderen Erfolg wünschen, die Einheitlichkeit der formalen Einrichtung wissenschaftlicher Veröffentlichungen, vor allem der Literaturverzeichnisse, befördern zu helfen.

Hans-Egon Hass 


\section{INHALT}

Geleitwort von Prof. Dr. Dr. Hans-Egon Hass, Freie UNIVERSITÄT BERLIN

VORWORT

IX

EINLEITUNG: Die SORgfältige Materialsammlung ALS wichTIGE VORARBEIT FÜR DIE MANUSKRIPTGESTALTUNG

A. Die Gestaltung des Manuskripts im allgemeinen

I. Die Gliederung $\quad 7$

II. Der Titel Io

III. Das Zitieren $\quad$ I3

IV. Die Anmerkungen 24

V. Das Literaturverzeichnis $\quad 27$

VI. Das Schriftbild 29

VII. Der ,Photodruck' und der Schriftsatz 35

B. Die Titelangaben von Schrifttum

Vorbemerkungen $\quad 42$

I. Umfang und Anordnung der Angaben 44

I. Selbständige Veröffentlichungen (außer Hochschulschriften)

Selbständige Schriften besonderer Art $\quad 56$

2. Hochschulschriften 66

3. Beiträge in Sammelwerken und Zeitschriften 69

II. Schrift und Schreibung 79

III. Die alphabetische Ordnung der Verfassernamen und der Sachtitel 80

IV. Die gekürzte Titelangabe und die Titelbeschreibung $\quad 86$

Anhang

I. Verzeichnis der wichtigsten Abkürzungen bibliographisch-technischer Ausdrücke

II. Korrekturzeichen und ihre Erklärung (DIN 165II) 93

III. Musterseiten $\quad 96$

$\begin{array}{ll}\text { REGISTER } & 98\end{array}$ 


\section{VORWORT}

Viele Dozenten klagen, daß die äußere Form von Seminar-, Staatsexamens-und Doktorarbeiten oft zu wünschen übrig lasse, $\mathrm{da} ß$ insbesondere die Literaturangaben vielfach unvollständig, irreführend, ja sogar falsch seien. Andererseits klagen die von solchen Vorwürfen Betroffenen und Bedrohten, daß es keine Anleitung für alle diese Dinge gebe. In der Tat finden sich zwar manche nützlichen Vorschläge für die Manuskriptgestaltung in einigen Einführungen in die Technik des wissenschaftlichen Arbeitens; aber einmal sind diese meist so allgemein gehalten, daß sie auf die besonderen Fragen, die sich bei einem philologischen Manuskript stellen, keine Antwort geben, zum anderen fehlen in allen diesen Einführungen genaue Anweisungen für die Titelangaben von Schrifttum. Gerade deren Gestaltung ist aber für den Germanisten ebenso wichtig wie schwierig.

Solche Einführungen sind z. B.:

Heyde, JohanNes Erich: Technik des wissenschaftlichen Arbeitens. Mit einem ergänzenden Beitrag Dokumentation von Heinz Siegel. 9., verb. u. erw. Aufl. - Berlin: Kiepert 1966.

Klieman, Horst: Anleitungen zum wissenschaftlichen Arbeiten.

Praktische Ratschläge u. erprobte Hilfsmittel. (5., verb. Aufl.;

r.-4. Aufl. u. d. T.: Praktikum der geistigen Arbeit.) - Freiburg: Rombach (1965).

Kröber, Walter: Kunst und Technik der geistigen Arbeit. 4., unveränd. Aufl. - Heidelberg: Quelle \& Meyer 1963.

SPIELER, Josef: Einführung und Anleitung zu wissenschaftlichem Denken und Arbeiten. Materie, Geist, Technik. Für Geistesarbeiter u. Studierende aller Fakultäten. - Olten: Walter (1946).

Auch die folgende Schrift, die in Anlehnung an die Stilmuster amerikanischer philologischer Zeitschriften gute Vorschläge für die Gestaltung gerade philologischer Manuskripte bringt, kann hinsichtlich der Titelangaben nicht befriedigen:

Standop, Ewald: Die Form der wissenschaftlichen Arbeit. 3., verb.

Aufl. - Dortmund: Lensing r965.

Standop folgt nämlich auch hierin den Richtlinien amerikanischer Zeitschriften, die jedoch keineswegs immer die nötige Klarheit ge- 
währleisten; besonders unübersichtlich ist die Nennung des Serientitels vor dem Erscheinungsvermerk des Stücktitels.

Wirklich einwandfrei und unmißverständlich sind Titelangaben nach bibliothekarischen Regeln. Ein etwa erhobener Einwand, sie würden auf diese Weise zu umfangreich und zu umständlich, wäre ungerechtfertigt und ließe sich leicht entkräften. Es wird keine Angabe gefordert, die nicht notwendig wäre, um eine Schrift eindeutig zu beschreiben und leicht auffindbar zu machen. Sodann ist die Befragung einer Schrift nach einem bestimmten Schema viel weniger umständlich als das tastende Suchen, welche Angaben des Titelblattes wohl in das Literaturverzeichnis aufgenommen werden müssen. Und schließlich gehört es doch zur geistigen Aneignung eines $\mathrm{Bu}-$ ches, daß jemand, der es benutzt und zitiert, auch weiß, welche Stellung es in derWelt der Bücher einnimmt, wie es sich etwa zu anderen Ausgaben, zu anderen Auflagen, zu anderen Bänden einer Reihe verhält, und daß er auch die Mühe nicht scheut, wichtige Angaben, die auf dem Titelblatt fehlen, aus anderen Quellen in Erfahrung zu bringen und zu ergänzen.

Es sei darauf hingewiesen, daß der Deutsche Normenausschuß die wichtigsten bibliothekarischen Regeln dem Normblatt DIN I 505 Titelangaben von Scbrifttum zugrunde gelegt hat. Ihnen folgen ferner grundlegende und vorbildliche Bibliographien unseres Faches, z. B. der Jabresbericht für deutsche Sprache und Literatur (Bd I. Bibliographie 1940-1 945. Berlin: Akademie-Verl. 1960), das Referatenorgan Germanistik (Jg. I ff. Tübingen: Niemeyer I960 ff.) sowie seit einiger Zeit auch Goedekes Grundriß zur Geschichte der deutschen Dicbtung (vgl. Hans Fromm: Germanistische Bibliographie seit 1945. Theorie u. Kritik. Stuttgart:Metzler 1960. (Referate aus der DVjs.) S. 40, Anm. 44). Deshalb sollten jedenfalls germanistische Arbeiten das gleiche tun.

Obwohl auch die Klarheit der Gliederung und die sorgfältigeWiedergabe von Zitaten nicht nur Formfragen sind, wollen die Ausführungen unseres ersten Teils weniger als Regeln denn als Vorschläge verstanden sein. Sie wenden sich vor allem an den, der noch keine festen und sicher gehandhabten Gewohnheiten der Manuskriptgestaltung hat. Sie wollen nicht schulmeistern, obwohl sie selten verschiedene Möglichkeiten zur Wahl stellen. Wir glauben, daß ihre Befolgung ein formal einwandfreies und ansprechendes Manuskript gewährleistet. 
Grundlage für die Vorschläge zur Manuskriptgestaltung waren, soweit es sich nicht um allgemein verbreitete Gepflogenheiten handelt, das Merkblatt für Doktoranden, das die Kommission für Dissertationsfragen des Vereins Deutscher Bibliothekare im Jahre 1956 entworfen hat, sowie die Normblätter DIN 5008 Regeln für Maschinenschreiben und DIN I422 Technisch-wissenschaftliche Veröffentlicbungen. Ricbtlinien für die Gestaltung. Dankbar wurden auch die schon genannte Schrift von STANDOP und das von William RILEy Parker zusammengestellte $M L A$ Style Sheet benutzt.

Alle Normblätter liefert der Beuth-Vertrieb in Berlin und Köln. Die Arbeit von Parker erschien in einer revidierten Auflage I960 bei der Modern Language Association of America in New York.

Grundlegend für die Normung von Titelangaben, die wir im zweiten Teil unserer Schrift (S. 42 ff.) behandeln, sind die sogenannten "Preußischen Instruktionen " (Instruktionen für die alphabetischen Kataloge der preußischen Bibliotheken vom ro. Mai I899, 2. Ausg. in der Fassung vom ro. August 1908). Sie waren immer wieder zu befragen; ihnen sind auch die Vorschriften für die alphabetische Ordnung der Namen und der Sachtitel (s. S. 80) entnommen. Zur Klärung einer Reihe von Einzelfragen war der Kommentar zu den Instruktionen von HERManN Fuchs sehr hilfreich. - Die Instruktionen betreffen in erster Linie die Anlage von Katalogkarten. Wie sie sich auf die Gestaltung bibliographischer Titelangaben auswirken, lehren die neueren deutschen Allgemeinbibliographien. Die Vorschriften des Normblattes DIN I 505 Titelangaben von Scbrifttum. Richtlinien wurden verglichen.

Instruktionen für die alphabetischen Kataloge der preußischen Bibliotheken vom Io. Mai I899. 2. Ausg. in der Fassung vom ı. Aug. I908. Unveränd. Nachdr. - Wiesbaden: Harrassowitz $196 \mathrm{r}$.

Fuchs, Hermann: Kommentar zu den Instruktionen für die alphabetischen Kataloge der Preußischen Bibliotheken. 2., durchges. Aufl. - Wiesbaden: Harrassowitz 1958.

Uber die Allgemeinbibliographien unterrichtet:

RAABE, PAUL: Einführung in die Bücherkunde zur deutschen Literaturwissenschaft. 5., verb. Aufl. - Stuttgart: Metzler 1966. (Sammlung Metzler.) S. 3 Iff. (besonders die Titel I26-1 32.) 
Neben den gedruckten Unterlagen durfte ich mich für die folgenden Ausführungen auch mancherlei mündlicher Hilfen dankbar bedienen. Vor allem haben die Herren Dr. KLAus Kanzog und Dr. Rainald Stromeyer die Arbeit durch wertvolle Hinweise gefördert. Herrn MARTin MACHATZke verdanke ich neben anderen Anregungen die Mitteilung der auf S. 42 zitierten Stelle des Herder-Heyne-Briefwechsels.

G. B.

\section{Zur dritten Auflage}

Dank dem großzügigen Entgegenkommen des Verlages konnte der Anhang erweitert werden. Zwei Musterseiten veranschaulichen die für die Gestaltung des Schriftbildes gegebenen Empfehlungen. Ferner wurde mit freundlicher Genehmigung des Deutschen Normenausschusses die Übersicht über die Korrekturzeichen nach DIN I6 II aufgenommen, damit der Benutzer der vorliegenden Schrift in ihr alles für die Manuskriptgestaltung und Drucklegung Wissenswerte vereint findet. Der Abschnitt über die gekürzten Titelangaben (S. 86ff.) wurde ohne Änderung im Grundsätzlichen etwas weiter ausgeführt. Sachlich neu ist auf S. 57 die Empfehlung, bei Übersetzungen den Originaltitel, wenn er in der Übersetzung angegeben ist, gemäß der Regel auf S. 46 in runden (statt eckigen) Klammern aufzuführen.

G. B.

\section{Zur vierten Auflage}

Die Literaturangaben und, sofern sinnvoll, auch die Titelbeispiele wurden auf den neuen Stand gebracht. Der Abschnitt über die Anführung von Zeitungsartikeln (S. 77 f.) bringt eine sachliche Änderung: die Empfehlung, auch die Nummer der zitierten Zeitung zu nennen. Herrn Peter Heilmann vom Institut für Publizistik an der Freien Universität Berlin verdanke ich den Hinweis auf die hierfür maßgebende neuere Praxis der publizistischen Dokumentation.

G. B. 